Case report

\title{
A case report of COVID-19 associated pulmonary mucormycosis
}

\author{
Nariman Khan ${ }^{* 1}$, Christina G. Gutierrez ${ }^{2,3}$, David Villafuerte Martinez ${ }^{4}$, Kevin C \\ Proud $1,4,5$
}

${ }^{1}$ UT Health San Antonio, Joe R. and Terry Lozano Long School of Medicine, Department of Internal Medicine, San Antonio, Texas, USA; 'University Health System, Pharmacotherapy and Pharmacy Services, San Antonio, Texas, USA; ${ }^{3}$ UT Health San Antonio, Pharmacotherapy Education and Research Center, San Antonio, Texas, USA; ${ }^{U}$ UT Health San Antonio, Joe R. and Terry Lozano Long School of Medicine, Department of Medicine, Division of Pulmonary Diseases and Critical Care Medicine, San Antonio, Texas, USA; ${ }^{5}$ South Texas Veterans Health Care System, Texas, USA

\begin{abstract}
Hospitalized patients with SARS-CoV-2 (severe acute respiratory syndrome coronavirus 2) are at risk for developing secondary fungal infections due to greater incidence of preexisting comorbidities and exposure to iatrogenic factors such as corticosteroid use. We present the case of a 44-year-old Hispanic female discovered unresponsive in her home that was found to have severe hyperglycemia with comorbid COVID-19 (coronavirus disease 2019) associated pneumonia. The patient was intubated and treated with several broad-spectrum antibiotics, remdesivir, and corticosteroids but had little improvement in her clinical status. Bronchoscopy was performed and revealed multiple necrotic lesions in the lungs. Endobronchial biopsy and bronchoalveolar lavage samples revealed pauciseptated hyphae consistent with zygomycetes. The patient was treated with multiple antifungals including voriconazole, micafungin, and amphotericin B. However, despite maximal medical therapy, the patient perished. This case highlights that clinicians must carry a high degree of suspicion and a low threshold to begin treatment for Mucor in diabetics and other immunosuppressed patients.
\end{abstract}

Keywords: SARS-CoV-2; COVID-19; mucormycisis; diabetes melitus; thoracic CT; remdesivir

\section{Introduction}

Mucormycosis is a rare infection seen primarily in patients with immunocompromise including uncontrolled diabetes, hematologic malignancies, and systemic corticosteroid use [1]. Patients diagnosed with severe COVID-19 pneumonia are at high risk for developing secondary fungal infections due to their hospitalized status, preexisting comorbidities, and treatment regimens consisting of steroids and oftentimes antibiotics.

Received: November 2020; Accepted after review: December 2020; Published: January 2021.

${ }^{*}$ Corresponding author: Nariman Khan, UT Health San Antonio, Joe R. and Terry Lozano Long School of Medicine, Department of Internal Medicine, 7703 Floyd Curl Dr, San Antonio, TX 78229, USA

Email: khan nariman@yahoo.com
COVID-19 pneumonia with superimposed Aspergillus infection has been welldocumented in literature since the dawn of the pandemic [2, 3]. However, there is limited data on co-infection with Mucor in critically-ill COVID-19 patients. Here we present a rare case of severe COVID-19 associated pneumonia complicated by invasive pulmonary mucormycosis.

\section{Case report}

A 44-year-old Hispanic woman with type II diabetes mellitus and no prior history of tobacco use was admitted to the hospital after being found unresponsive in her home. On arrival she had a temperature of $36.6 \mathrm{C}$ degrees, heart rate 
of $126 \mathrm{bpm}$, respiratory rate of 26 , blood pressure of $160 / 90$, and an oxygen saturation of $78 \%$ on room air. Her physical exam was remarkable for a Glasgow coma score of 3 , dry mucous membranes, and clear lung sounds to auscultation bilaterally. She was intubated upon arrival. Laboratory studies revealed a white blood cell count of 13000, CRP 412, blood glucose $997 \mathrm{mg} / \mathrm{dl}$, serum sodium 160 $\mathrm{mmol} / \mathrm{L}$, serum creatinine of $3.72 \mathrm{mg} / \mathrm{dL}$, and serum bicarbonate 11 . Her arterial blood gas showed $\mathrm{pH}$ of 7.08, $\mathrm{PaCO} 237.1$, and $\mathrm{PaO} 2$ of 96 on $60 \%$ fraction of inspired $\mathrm{O} 2$ (FiO2) and she tested positive for SARS-CoV-2 by RTPCR. COVID-19 specific labs were drawn and revealed elevated D-dimer to 1393 and normal values for troponin, BNP, procalcitonin, INR, AST/ALT, and ferritin. The patient was started on an insulin drip for her hyperglycemic syndrome. Her COVID-19 pneumonia was treated with 5 days of both remdesivir $100 \mathrm{mg}$ IV daily and methylprednisolone $30 \mathrm{mg}$ IV twice a day. Due to the critical presentation of the patient, empiric treatment for a possible superimposed bacterial pneumonia was also initiated on hospital day 1 with the broadspectrum antibiotics cefepime and vancomycin.
The patient's metabolic derangements improved during the first 3 days of her ICU stay, however her respiratory status worsened and she developed acute respiratory distress syndrome associated with her COVID-19 pneumonia. She required optimization of her ventilatory parameters along with rescue therapies including neuromuscular blockade and prone positioning by hospital day 4 . Tracheal aspirate cultures were drawn at this time and revealed Pseudomonas aeruginosa and Aspergillus flavus. The patient was started on voriconazole $250 \mathrm{mg}$ IV twice daily and switched to piperacillin/tazobactam $4.5 \mathrm{~g}$ IV every eight hours based on susceptibilities.

Computed tomography of the chest was obtained on hospital day 8 due to worsening hypoxemia and revealed diffuse ground glass opacities (GGO) and multiple cavitary lesions measuring up to $2.1 \mathrm{~cm}$ in the right middle and upper lobes (Figures 1 and 2). Micafungin 150 mg IV daily was added to the patient's treatment regimen on hospital day 9 for invasive pulmonary aspergillosis given her poor response to treatment.

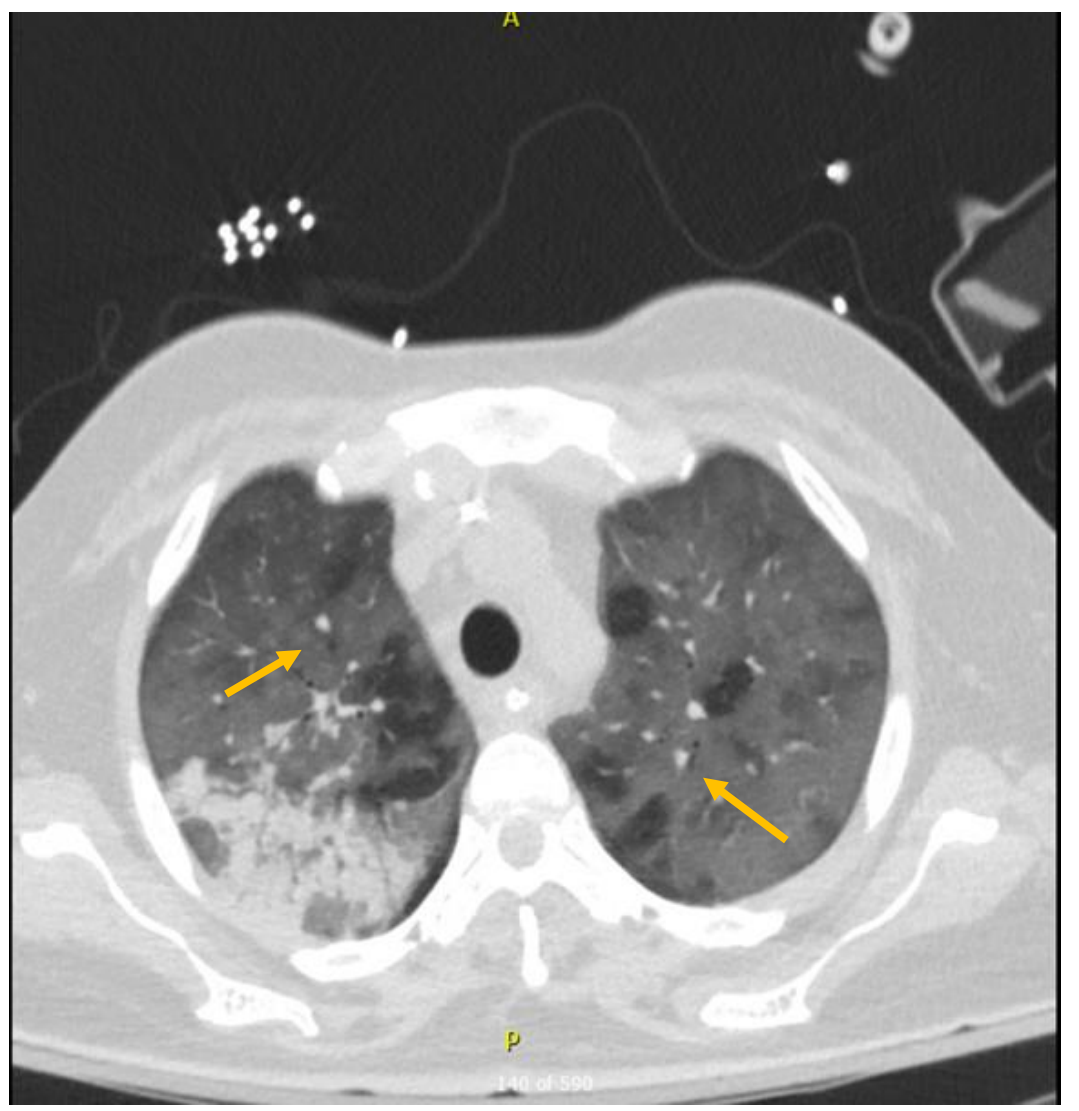

Fig. 1. Computer tomography of the chest, axial view: diffuse GGO (arrows) throughout both lung fields. 


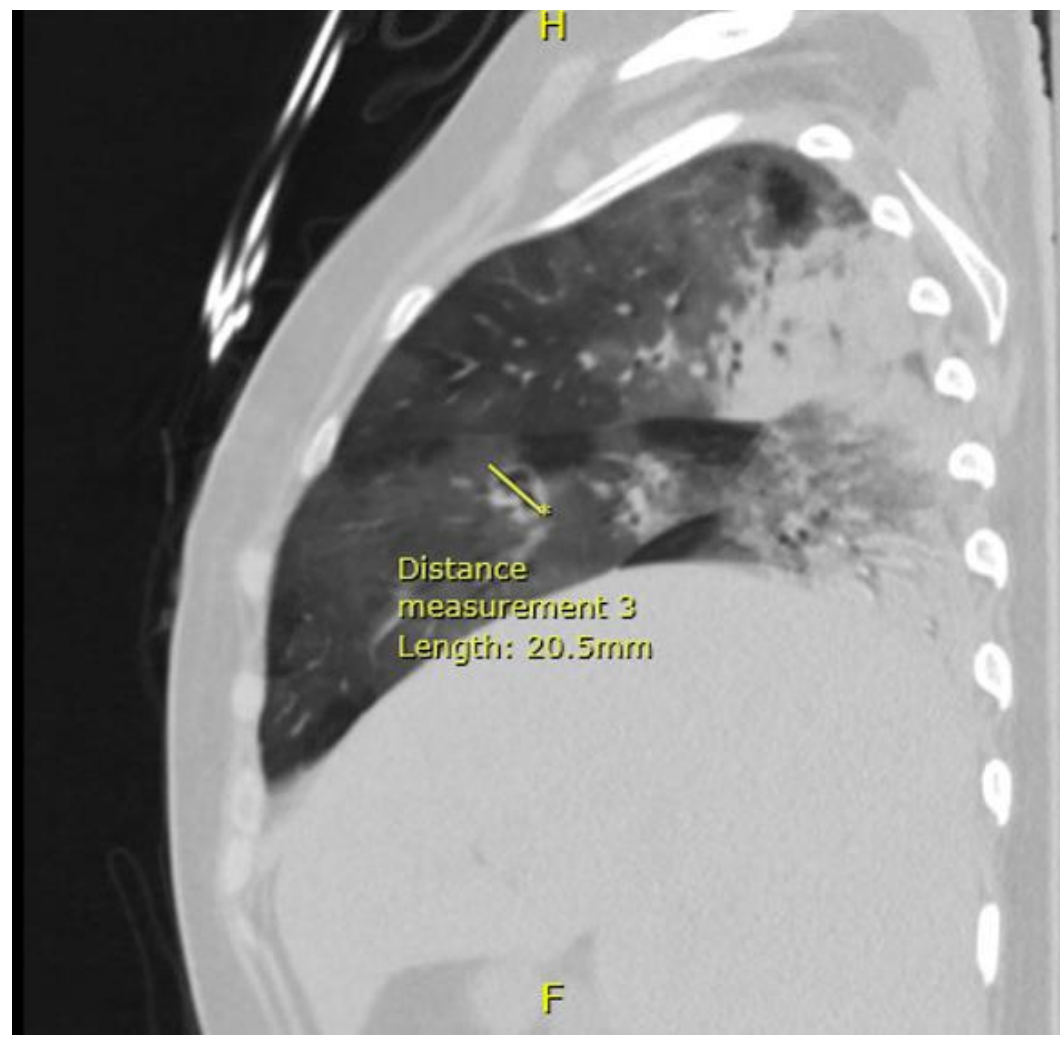

Fig. 2. Computer tomography of the chest, sagittal view: approximately $2 \mathrm{~cm}$ cavitary lesion in right middle lobe

Bronchoscopy was performed on hospital day 13 due to worsening ventilation/perfusion ratio to less than 100 and lack of response to treatment. Multiple gray necrotic-appearing mucosal lesions were visualized bilaterally with near complete destruction of the left upper lobe and lingula. The lesions could be easily detached from the endobronchial wall and were non-friable. Endobronchial biopsy and bronchoalveolar lavage (BAL) were performed on the necrotic appearing lingula and Grocott's methenamine silver stain on the sample revealed pauciseptated hyphae consistent with zygomycetes. Culture results were significant for isolated Candida albicans, glabrata, and krusei along with Aspergillus flavus and niger. The patient's hemoglobin A1c was then checked and found to be 13. Given the endobronchial destruction on bronchoscopy, the histopathologic findings, and evidence of poorly controlled diabetes, a clinical diagnosis of pulmonary mucormycosis was made and the patient was then started on treatment with liposomal amphotericin B $5 \mathrm{mg} / \mathrm{kg} /$ day.

Despite aggressive therapy, the patient's condition continued to decline. She required continuous renal replacement therapy and was deemed not to be a candidate for extracorporeal membrane oxygenation given her devastating pulmonary disease and multiorgan dysfunction. The patient expired on hospital day seventeen.

\section{Discussion}

Secondary infections with invasive pulmonary aspergillosis are well documented in critically ill patients with COVID-19 [2, 3]. However, there have only been isolated case reports of Mucor in COVID-19 patients, including one by Mehta et al. of a 60-year-old diabetic patient with rhino-orbital mucor confirmed by nasal biopsy culture. The patient eventually developed a severe cytokine storm and died from shock [4]. Another example presented by Werthman-Ehrenreich documents the case of a 33-year-old diabetic woman in ketoacidosis with concomitant COVID-19 and biopsy culture proven rhinoorbital-cerebral mucormycosis. The patient was eventually transitioned to comfort care due to 
extensive fungal neurological involvement and poor prognosis [1]. Finally, Placik et al. document the case of a healthy 49-year-old man with COVID-19 that eventually developed a tension pneumothorax with a bronchopleural fistula. Cardiothoracic surgery performed a thoracotomy for fistula repair and intraoperatively found an empyema that grew Rhizopus species on culture. This patient also succumbed to his illness [5]. Mucormycosis without concomitant COVID-19 infection has a mortality rate ranging from $40-80 \%$ [6]. This mortality rate appears to reach $100 \%$ when these two infections co-occur based on documented cases in literature thus far.

Diabetes remains the greatest risk factor for mucormycosis, but more recently there has been a rise in cases among patients with immunosuppressive conditions. Diabetic patients typically present with rhino-cerebral disease whereas pulmonary mucormycosis is better documented in immunosuppressed patients such as transplant recipients [7]. Our patient was at very high risk for developing an invasive fungal infection due to her uncontrolled diabetes, hospitalized status, and systemic corticosteroid use as part of her treatment regimen. Mucorales infections are characterized by rapid progression and angioinvasion resulting in thrombosis and extensive tissue necrosis. Pulmonary mucormycosis manifests with persistent fever, cough, and dyspnea, while rhino-cerebral mucormycosis presents with fever, unilateral facial swelling, headache, sinus congestion, and dark lesions on the nasal bridge and inner mouth [8]. Our patient exhibited persistent fever that prompted further diagnostic workup yielding diagnosis.

Imaging findings of mucormycosis are nonspecific as they can present as a solitary nodule, lobar consolidation, or a cavitary lesion depending on the severity of the infection [9]. Hammer et al. researched the CT features of pulmonary mucormycosis in 30 patients with definite or probable disease. They found that the reverse halo sign, which is a GGO surrounded by a denser consolidation of crescentic shape was found in 67 percent of their patients at some point in their disease course. They also found that 53 percent of patients had a large perilesional halo and 87 percent had peripheral distribution of their lung lesions [10]. However, CT findings alone cannot diagnose pulmonary mucormycosis as some findings such as the reverse halo sign may also be found in invasive aspergillosis and COVID19 pneumonia. Our patient's CT chest showed diffuse GGO and multiple cavitary lesions which were nonspecific and likely due to her multiorganism infection with COVID-19, mucormycosis, and aspergillosis. Similarly, laboratory testing such as the beta-D-glucan assay and serum galactomannan antigen can aid in determining the presence of a fungal infection, but neither are associated with Mucorales. Thus, diagnosis of mucormycosis requires a multifaceted approach consisting of clinical observations, radiographic and histopathologic findings, and fungal culture analysis. It is important to note that cultures of mucormycosis often produce no growth and in these situations proper clinical context is necessary to establish a diagnosis [11].

Our patient was treated with voriconazole, micafungin, and liposomal amphotericin B due to her multi organism culture growth. Aggressive source control with surgical debridement is the cornerstone of management. When surgical debridement is not an option due to high surgical mortality risk, as was the case for our patient, systemic treatment with high dose liposomal amphotericin B is the standard of care. Our patient was placed on liposomal amphotericin B $5 \mathrm{mg} / \mathrm{kg} /$ day, however higher doses of up to $10 \mathrm{mg} / \mathrm{kg} /$ daily have been associated with increased response to treatment and our patient may have benefited from rapid dose escalation [12]. However, she had significant acute kidney injury throughout her hospitalization and likely would not have tolerated maximal dosing.

Combination of amphotericin B with either posaconazole or echinocandins is not currently recommended by any major treatment guidelines. However, there are studies that show improved survival in mice and human subjects with mucormycosis treated with a combination of echinocandin and amphotericin therapy. Ibrahim et al. induced neutropenia or DKA in mice then infected them with mucormycosis. They found that mice treated with micafungin or anidulafungin with liposomal 
amphotericin B had improved survival compared to monotherapy with any agent alone [13]. Additionally, a retrospective study on 41 patients with rhino-orbital-cerebral mucormycosis compared the effects of polyene (amphotericin B lipid complex, liposomal amphotericin B, or amphotericin B deoxycholate) plus caspofungin therapy versus polyene monotherapy on survival time. The authors found that combination therapy was associated with greater survival time and improved outcomes at 30 days post discharge [14]. Combination therapy was utilized in this patient with the hope of improving her outcome given that she was not a surgical candidate.

\section{Conclusion}

As COVID-19 cases continue to rise, increased surveillance for co-infections with

\section{References}

1. Werthman-Ehrenreich A. Mucormycosis with orbital compartment syndrome in a patient with COVID-19. Am J Emerg Med 2020; 10.1016/j.ajem.2020.09.032

2. Mohamed A, Rogers TR, Talento AF. COVID-19 associated invasive pulmonary aspergillosis: Diagnostic and therapeutic challenges. J Fungi (Basel) 2020; 6(3):115. doi: 10.3390/jof6030115. PMID: 32707965; PMCID: PMC7559350.

3. Koehler P, Cornely OA, Böttiger BW, et al. COVID-19 associated pulmonary aspergillosis. Mycoses 2020; 63(6):528-534. doi:10.1111/myc.13096

4. Mehta S, Pandey A: Rhino-orbital mucormycosis associated with COVID-19. Cureus 2020; 12(9):e10726. doi:10.7759/cureus.10726

5. Placik DA, Wesley TL, Nathan WM: Bronchopleural fistula development in the setting of novel therapies for acute respiratory distress syndrome in SARS-CoV-2 pneumonia. Radiol Case Rep 2020; 15(11):2378-2381. 10.1016/j.radcr.2020.09.026

6. Cornely OA, Alastruey-Izquierdo A, Arenz D, et al. Global guideline for the diagnosis and management of mucormycosis: an initiative of the European Confederation of Medical Mycology in cooperation with the Mycoses Study Group Education and Research unique organisms should be continued. Diagnosis of mucormycosis is difficult because it frequently requires invasive measures such as bronchoscopy. Clinicians should be aware of this risk in COVID pneumonia patients with persistent fever and hypoxemia. They must have a high degree of suspicion and a low threshold to begin treatment for Mucor, particularly in diabetics and immunosuppressed patients.

\section{Consent}

Written informed consent was obtained from the patient's relatives for publication of this case report and accompanying images. A copy of the written consent is available for review by the Editor-in-Chief of this journal.

\section{Competing interests}

The authors declare that they have no competing interests.
Consortium. Lancet 2019; 19(12):e405-e421 doi: 10.1016/S1473-3099(19)30312-3

7. Jeong $\mathrm{W}$, Keighley $\mathrm{C}$, Wolfe $\mathrm{R}$, et al.: The Epidemiology and Clinical Manifestations of Mucormycosis: A Systematic Review and MetaAnalysis of Case Reports. Clin Microbiol Infect 2019; 25(1):26-34. 10.1016/j.cmi.2018.07.011

8. Symptoms of Mucormycosis. (2020): https://www.cdc.gov/fungal/diseases/mucormyc osis/symptoms.html Accessed: December 30, 2020

9. Pulmonary Mucormycosis. (2020): https://radiopaedia.org/articles/pulmonarymucormycosis?lang=us Accessed: December 30, 2020

10. Hammer MM, Madan R, Hatabu H: Pulmonary mucormycosis: radiologic features at presentation and over time. $A J R A m \mathrm{~J}$ Roentgenology 2018; 210(4):742-747. 10.2214/AJR.17.18792

11. Mucormycosis (zygomycosis). (2020): https://www.uptodate.com/contents/mucormyco sis-zygomycosis/print Accessed: December 30, 2020

12. Lanternier F, Poiree $S$, Elie $C$, et al.: Prospective pilot study of high-dose (10 $\mathrm{mg} / \mathrm{kg} /$ day $)$ liposomal amphotericin B (L-AMB) for the initial treatment of mucormycosis. I Antimicrob Chemother 2015; 70(11):3116-3123. 10.1093/jac/dkv236 
13. Ibrahim AS, Gebremariam T, Fu Y, Edwards JE Jr, Spellberg B: Combination echinocandinpolyene treatment of murine mucormycosis. Antimicrob Agents Chemother 2008; 52(4):1556-1558. 10.1128/AAC.01458-07
14. Reed C, Bryant R, Ibrahim AS, et al. Combination polyene-caspofungin treatment of rhino-orbital-cerebral mucormycosis. Clin Infect Disease 2008; 47(3):364-371. 10.1086/589857 